\title{
12
}

\section{Control Optimisation and Parametric Design}

\author{
J.V. Ringwood
}

Centre for Ocean Energy Research, Maynooth University, Maynooth, Ireland

\subsection{INTRODUCTION}

In this chapter, the use of wave energy converter (WEC) models for optimization and control will be investigated. Since the choice of modelling strategy, and the nature/structure of the model itself, is very much influenced by the application for which the model is used, significant consideration will be given to the modelling requirements which are particular to WEC control (and control of WEC arrays) and the constraints or characteristics relevant to optimization needs.

A variety of WEC model use is explored throughout this book. In many cases, the focus is on developing a model that can accurately predict device responses, or allow device developers to assess the performance of a device under a specific set of sea conditions. Alternative model uses include the desire to assess the performance of various WEC control strategies in simulation, or to assess the likely power production from a WEC device under a specific set of sea conditions (Chapter 13). In this chapter, however, the use of a WEC model either to evolve a control design algorithm or to include a WEC simulation or evaluation model within an optimization loop, places demanding computational and complexity limitations on allowable WEC models. For the control case, two requirements need to be met:

(a) There needs to be an algebraic route between the model parameters and the control parameters, and

(b) The derived controller must be able to execute in real time.

Item (a) places a complexity limit on the WEC model, while (b) also places a computational complexity limit. As a very rough guide, WEC devices designed to operate in Atlantic seas are likely to experience waves with periods around $10 \mathrm{~s}$, suggesting that the sampling rate for such a WEC controller would operate in the region of $10 \mathrm{~Hz}$, giving $0.1 \mathrm{~s}$ maximum for controller calculations. For use in numerical optimization loops (eg, optimizing the geometry of a device or array layout) the WEC/array models do not need to compute in real time, but since the optimization is likely to take a considerable number of iterations, and hydrodynamic parameters may need to be recomputed at each iteration, some economy needs to be achieved in the model complexity if optimization results are to be returned in a reasonable time. 
In some cases, the WEC control algorithms are not simply algebraic transformations of the WEC model, but themselves involve numerical optimization routines which use a WEC model, in tandem with a numerical objective function; for example, see Hals et al. (2011b), Cretel et al. (2011), Brekken (2011), and Bacelli and Ringwood (2015). Though such control algorithms have a high computational overhead, they have the advantage of being able to take device constraints (displacement, power takeoff (PTO) force, etc.) into account.

\subsection{CONTROL OF WECs}

In this section, the control of WECs and arrays of WECs is considered. Control refers to any device or algorithm used to alter the behaviour of a WEC or an array of WECs. There are multiple levels of WEC and WEC array behaviour at which control can be applied. In general, and in keeping with the spirit of this book, a model-based approach to control will be considered here, though we note that model-free approaches (for example, extremumseeking control, Hals et al., 2011a) are also possible.

While the word 'control' used in connection with WECs and WEC arrays is consistent with the general use of the word in broader control applications, in that the behaviour of a system is altered, the control objective in the wave energy case diverges from traditional control objectives. Typically, traditional control loops are used to follow a setpoint (the servomechanism problem), or minimize the variance of a system output variable (the regulator problem). In both cases, the tendency is to drive the output to a certain demanded value, which is often constant. Two characteristics, which traditional control systems depend on, include:

The use of feedback, which has the added advantage of a reduction in the sensitivity of the closed-loop system to variation in the open-loop characteristics, including the system model, as well as providing set-point tracking, and

Forcing the system variables to a (relatively) constant value, which helps to validate the use of a linearized system model (for control design and simulation) about a fixed operating point or well-defined small region.

In contrast, the objective in WEC control is the maximization of captured energy, via a performance function of the form:

$$
J=\int_{0}^{T} v(t) f_{p t o}(t) d t,
$$

where $v(t)$ is the device velocity in the degree(s) of freedom harnessed by the PTO and $f_{u}(t)$ the corresponding PTO force(s) exerted, subject to device physical constraints. Maximization of Eq. (12.1) is usually achieved by maximizing the motion of the device, which makes it difficult to justify linearization about an equilibrium point (usually the still water level is chosen). In addition, WEC control generally relies on feedforward control to generate optimal device velocity or PTO force setpoints (see Fig. 12.1) so that the attractive sensitivity properties of feedback systems cannot be relied on.

As a result, WEC control is a challenging problem, made considerably more challenging by the difficulty of obtaining lumpedparameter models that lend themselves to control design.

\subsubsection{Control Effectors}

Since wave energy PTO systems typically involve a number of changes of energy form, there can be a variety of ways to implement the required PTO force, in order to achieve the desired device velocity that maximizes Eq. (12.1). Fig. 12.2 shows a number of possible variables that can be 


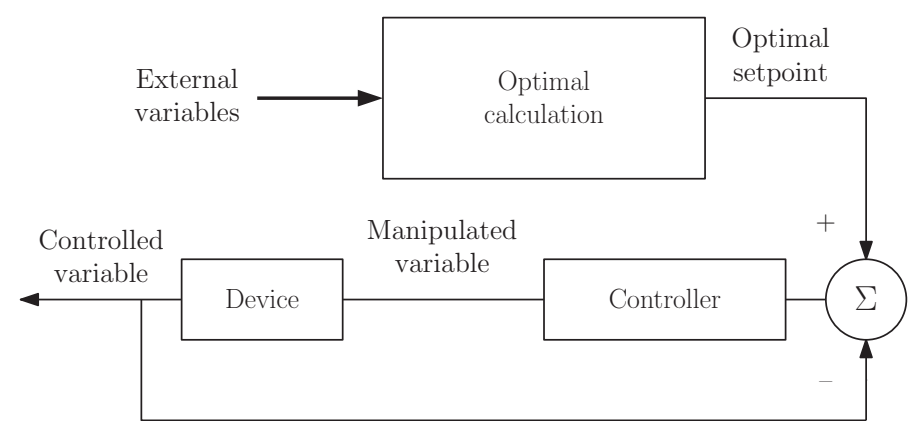

FIG. 12.1 Hierarchical control structure, showing the optimal setpoint (feedforward) calculation and the servomechanism section that adjusts the PTO so that the optimal (force/velocity) setpoint is achieved.

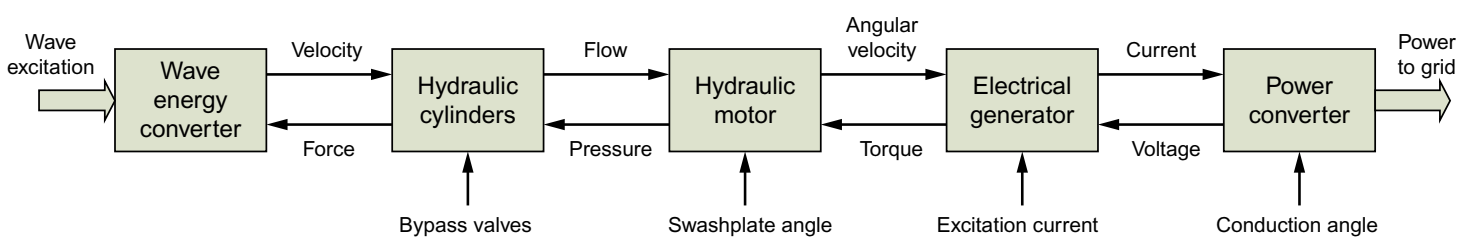

FIG. 12.2 Wave energy PTO system components and potential control inputs. In general, only one of these control inputs is used by the energy-maximizing control.

manipulated to control the PTO force which influences the WEC device motion, including:

- Hydraulic motor swashplate angle,

- Generator excitation current, and

- Power converter conduction angle.

In general, only one of these inputs is used, though consideration of efficiency of the various included PTO components might suggest that some combination might be beneficial, in particular circumstances. A manipulated control variable, for WECs where multiple hydraulic cylinders or (linear) electrical generators are used, could be the number of cylinders/generators employed either on a wave-to-wave basis, or for significant changes in sea-state. Hydraulic bypass valves could be used to deactivate hydraulic cylinders, while nonrequired linear generators could be electrically short-circuited. A final control possibility is that of pumpable water ballast, which can be used to alter the WEC inertia and therefore change its resonant frequency. An example study using ballast control for a bottom-hinged flap was performed by Qiu et al. (2013). However, the use of water ballast as a control input has limitations, including maximum pumping rate (determined by pump size) and the energy cost of moving water ballast.

It should be borne in mind that, for each PTO component of Fig. 12.2 used, a model is required. Ultimately, if we require to simulate a complete WEC system, a model of the full system from free surface elevation variations to the required output (eg, electrical power), taking into account intermediate inputs such as control inputs, is required. Similarly, a model-based WEC control algorithm will require a full model of the system from (usually) a measure of either the excitation force or free surface elevation to the control input point. In addition, control algorithms may also require a measure of an objective function, usually involving output power. In this book, the focus is on hydrodynamic modelling, so this chapter will also focus on hydrodynamic aspects of modelling and control. 


\subsubsection{Fundamental Control Results}

With reference to the frequency-domain models developed in Chapter 2, we can recall the energy maximization problem by considering the force-to-velocity model of a WEC as (Falnes, 2002):

$$
\frac{V(\omega)}{F_{e x}(\omega)+F_{u}(\omega)}=\frac{1}{Z_{i}(\omega)},
$$

where $Z_{i}(\omega)$ is termed the intrinsic impedance of the system. In Eq. (12.2), $V(\omega), F_{e x}(\omega)$, and $F_{u}(\omega)$ represent the Fourier transform of the velocity, $v(t)$, excitation force, $f_{e x}(t)$ and control force $f_{\text {pto }}(t)$, respectively. Note that, in the following, unless stated otherwise, the Fourier transform of time-domain signals or functions will be denoted by the corresponding capital letter, namely $X(\omega) \triangleq \mathcal{F}\{x(t)\}$.

The intrinsic impedance, $Z_{i}(\omega)$, of the model in Eq. (12.2) is specified as (refer to Falnes (2002) for the full derivation):

$$
Z_{i}(\omega)=B_{r}(\omega)+\mathcal{J} \omega\left[M+M_{a}(\omega)-\frac{K_{b}}{\omega^{2}}\right],
$$

where $B_{r}(\omega)$ is the radiation resistance (real and even) and $M_{a}(\omega)$ is the added mass.

The model in Eq. (12.2) allows the derivation of conditions for optimal energy absorption and the intuitive design of the energy maximizing controller in the frequency domain (Falnes, 2002) as:

$$
Z_{\text {PTO }}(\omega)=Z_{i}^{*}(\omega),
$$

where ()$^{*}$ denotes the complex conjugate. The choice of $Z_{P T O}$ as in Eq. (12.4) is referred to as complex conjugate control, but many (especially electrical) engineers will recognize this choice of $Z_{P T O}$ as the solution to the impedance matching problem represented by Fig. 12.3. The result in Eq. (12.4) has a number of important implications:

- $Z_{P T O}(\omega)$ is frequency dependent, implying that there is a different optimal impedance for

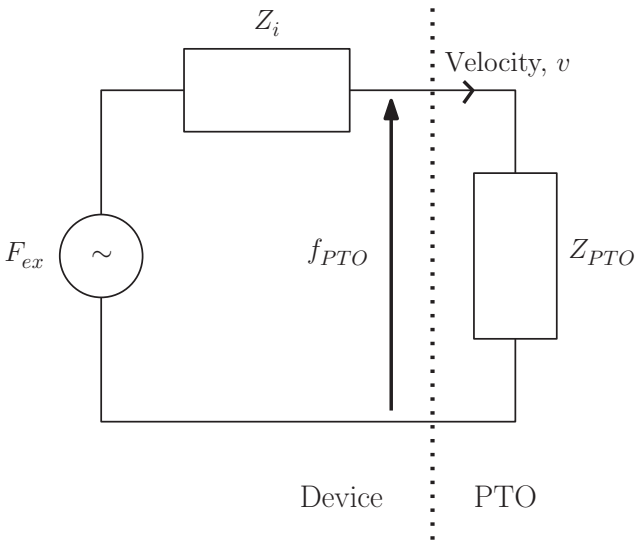

FIG. 12.3 Impedance matching for a wave energy device, directly analogous to its electric circuit counterpart.

each frequency-which raises the issue of how to specify $Z_{P T O}(\omega)$ for irregular seas containing a mixture of frequencies?

- Since the radiation impulse response, $h_{r}(t)$, is causal, $h_{c}(t)=\mathcal{F}^{-1}\left(Z_{P T O}(\omega)\right)$ is anticausal, requiring future knowledge of the excitation force. While this is straightforward for the monochromatic case (single sinusoid), it is more problematic for irregular seas. The issue of forecasting random seas is dealt with in Section 12.2.5.

- Since force and velocity can have opposite signs in Fig. 12.3, the PTO may supply power for some parts of the sinusoidal cycle. This is akin to reactive power in power systems. Such a phenomenon places particular demands on PTO systems, not only in terms of the need to facilitate bidirectional power flow, but also that the peak reactive power can be significantly greater than active power (Shek et al., 2008; Zurkinden et al., 2013). The optimal passive PTO is provided by $Z_{P T O}=\left|Z_{i}(\omega)\right|$, which avoids the need for the PTO to supply power, but results in a suboptimal control.

- The optimal control in Eq. (12.4) takes no account of physical constraints in the WEC/PTO, where there are likely to be 
limitations on displacement or relative displacement, PTO force and there may be external constraints imposed by electrical grid regulations.

- In Eq. (12.3), $B_{r}(\omega)$ and $M_{a}(\omega)$ are usually normally calculated as nonparametric functions of $\omega$ (see Chapters 2, 3 and 4). In order to use the model-based impedance matching approach, it is usually required to parameterize $B_{r}(\omega)$ as a finite-order differential function (see Chapter 3), which effectively replaces the convolution integral in Cummins equation with a finite-order differential equation. The relationship between the radiation damping convolution kernel function $k(t)$ and $B_{r}(\omega)$ and $M_{a}(\omega)$ is provided by Ogilvie's relations (Ogilvie, 1964).

The condition in Eq. (12.4) can also be expressed in terms of an optimal velocity profile as:

$$
V^{\text {opt }}(\omega)=F_{e x}(\omega) /\left(2 R_{i}(\omega)\right),
$$

where $R_{i}=1 / 2\left(Z_{i}+Z_{i}^{*}\right)$ is the real part of $Z_{i}$. The condition in Eq. (12.5) is a condition on the amplitude of $V^{o p t}(\omega)$, with the restriction that $v^{o p t}(t)$ be in phase with $f_{e x}(t)$, since $R_{i}$ is a real (and even) function. This phase condition, considered separately, forms the basis for some simple WEC phase control strategies, such as latching (Falnes, 2002).

While the conditions of Eqs (12.4) and (12.5) specify the optimal device velocity profile, they don't specify how it might be achieved. Fig. 12.1 shows a hierarchical structure for WEC control, where the optimal velocity is calculated in the upper branch and the PTO force is used to achieve this velocity in the lower servo loop. A number of studies have documented lower-loop control strategies for WEC PTO systems, including solutions based on internal model control (IMC) (Fusco and Ringwood, 2013; Beirão et al., 2007) and proportional-integral-plus (PIP) control (Taylor et al., 2009). A robust control strategy, using a passivity-based controller, can also be used (Fusco and Ringwood, 2014). Finally, in some cases, an integrated high/lowlevel controller is employed as, for example, in Falcão (2007) for a two-body WEC with a hydraulic PTO system.

\subsubsection{Real-Time Model-Based WEC Control}

In this section, three different example control strategies are presented, with increasing complexity (and potential resulting performance) of WEC model employed. In general, the complexity of the control algorithm is broadly consistent with the complexity of the model employed, though the way in which the control problem is formulated is also a determinant of the control algorithm complexity. Note that, for real-time control, a time-domain WEC model is required. For example, model predictive control (MPC) (Li and Belmont, 2014) uses a simulation model of the system contained within an optimization loop, which maximizes the objective function (energy) with respect to the control signal (PTO force or velocity), resulting in significant computational requirements.

In the following, Section 12.2.3.1 describes a simple controller, which is loosely based on the system model, but potentially offers good robustness properties and can handle PTO amplitude constraints. The controller described in Section 12.2.3.2 essentially inverts the complete Cummins equation model (constraints are not considered), while the controller of Section 12.2.3.3 uses constrained numerical optimization to solve the WEC control problem.

\subsubsection{A Simple but Effective WEC Controller}

This controller, fully reported in Fusco and Ringwood (2013), effectively parameterizes the radiation damping dynamics by a constant, resulting in a controller which has a very simple parametric form. Consider Eq. (12.5), which calculates the optimal velocity profile as a 
(frequency-dependent) function of the excitation force. In the following, a nonoptimal approximation of reactive control is proposed, where the noncausality and constraints are handled in a simple, but effective, way. The essence of this algorithm is the assumption that $f_{e x}(t)$ is a narrow-banded harmonic process, defined by time-varying amplitude, $A(t)$, frequency, $\omega(t)$, and phase $\varphi(t)$ :

$$
f_{e x}(t)=A(t) \cos (\omega(t) t+\varphi(t)) ;
$$

the optimal reference velocity can then be generated from the following adaptive law:

$$
v_{\text {ref }}(t)=\frac{1}{H(t)} f_{e x}(t), \frac{1}{H(t)}=\frac{1}{2 R_{i}(\hat{\omega})},
$$

where the value of the constant $H(t)$ is calculated from the curve $1 / 2 B(\omega)$, based on a real-time estimate of the peak frequency of the wave excitation force. An on-line estimate of the frequency, $\hat{\omega}$, and amplitude, $\hat{A}$, is obtained with the extended Kalman filter (EKF) (Quine et al., 1995). Based on the narrow-banded assumption of Eq. (12.6), the excitation force can be expressed in complex notation as:

$$
f_{e x}(t)=\mathcal{R}\left\{A e^{\mathcal{J} \varphi} e^{\mathcal{J} \omega t}\right\}, \quad \hat{F}_{e x} \triangleq A e^{J \varphi},
$$

where $\hat{F}_{e x}$ is the complex amplitude of $f_{e x}(t)$.

As a consequence of the proportional reference-generation law in Eq. (12.7), the complex amplitude of the velocity, $\hat{V}$, and position, $\hat{U}$, can be expressed as:

$$
\begin{gathered}
\hat{V}=\frac{A}{H} e^{\mathcal{J} \varphi} \\
\hat{U}=\frac{\hat{V}}{\mathcal{J} \omega}=\frac{A}{\mathcal{J} \omega H} e^{\mathcal{J} \varphi} .
\end{gathered}
$$

Suppose that the vertical excursion of the WEC is limited to $\pm U_{\text {lim }}$ from equilibrium. From Eq. (12.10), the position constraint can be written as an equivalent velocity constraint:

$$
\hat{U}=\frac{\hat{V}}{\mathcal{J} \omega} \leq U_{\text {lim }} \Leftrightarrow|\hat{V}| \leq \omega U_{\text {lim }}
$$

and the following upper bound for the variable gain, $1 / H$, involving the amplitude and frequency of the excitation, can be derived from Eq. (12.9) as:

$$
\frac{1}{H} \leq \frac{\omega U_{l i m}}{A}
$$

The reference generation strategy, based on Eqs (12.5), (12.7), and (12.12), can therefore be modulated in order to keep the amplitude of the velocity within the bound specified in Eq. (12.11). A real-time estimate of the frequency, $\hat{\omega}$, and amplitude, $\hat{A}$, of the excitation, can be obtained through the EKF (Budal and Falnes, 1982; Fusco and Ringwood, 2010):

$$
\frac{1}{H(t)}=\left\{\begin{array}{l}
\frac{1}{2 R_{i}(\hat{\omega})}, \text { if } \frac{\hat{\omega} U_{l i m}}{\hat{A}}>\frac{1}{2 R_{i}(\hat{\omega})} \\
\frac{\omega U_{l i m}}{\hat{A}}, \text { otherwise. }
\end{array}\right.
$$

According to Eq. (12.13), when in the unconstrained region, the velocity is tuned to the optimal amplitude given by complex-conjugate control, as in Eq. (12.5). Otherwise, the maximum allowed velocity (lower than the optimal) is imposed, while keeping the velocity in phase with the excitation force. The control structure is illustrated in Fig. 12.4. Since the algorithm is only loosely based on the WEC model, it has relatively good robustness properties to model error. Lower loop control as illustrated in Figs 12.1 and 12.4 is performed using IMC (Morari and Evanghelos, 1989), while a robust servo controller has been developed in Fusco and Ringwood (2014). The simple but effective (SE) controller, when compared with a MPC in both wide- and narrow-banded seas, has a relative capture width (RCW) within about $10 \%$ of the MPC (see Fig. 12.5), and even outperforms the MPC for long wave periods in the low $H_{s}$ case. However, the simple controller has superior robustness to variations in $K_{b}$ and has a tiny 


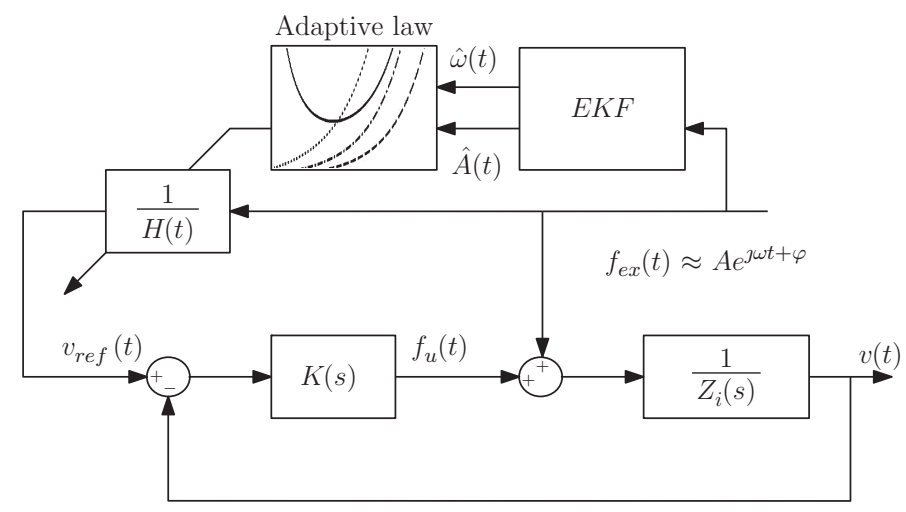

FIG. 12.4 Proposed control architecture for the simple/effective controller. The EKF effectively tracks the wave frequency and amplitude as in Eq. (12.6), while the $1 / H(t)$ block provides an adaptive feedforward gain to determine the optimal velocity profile. $K(s)$ regulates the PTO to ensure that the optimal velocity profile is achieved.
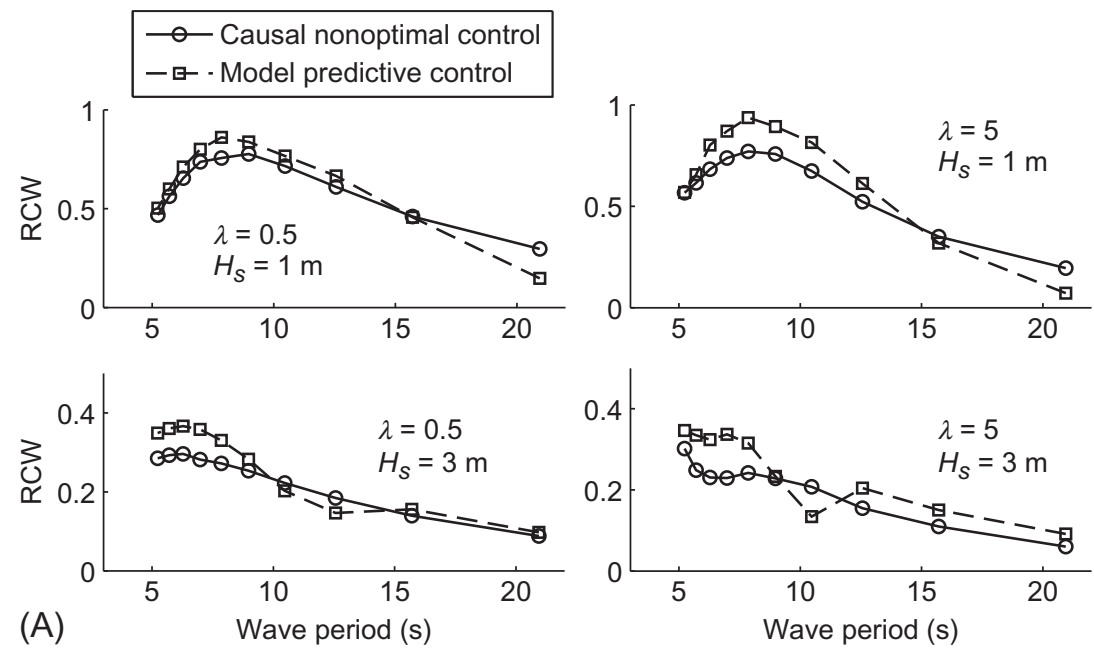

(A) Wave period (s)
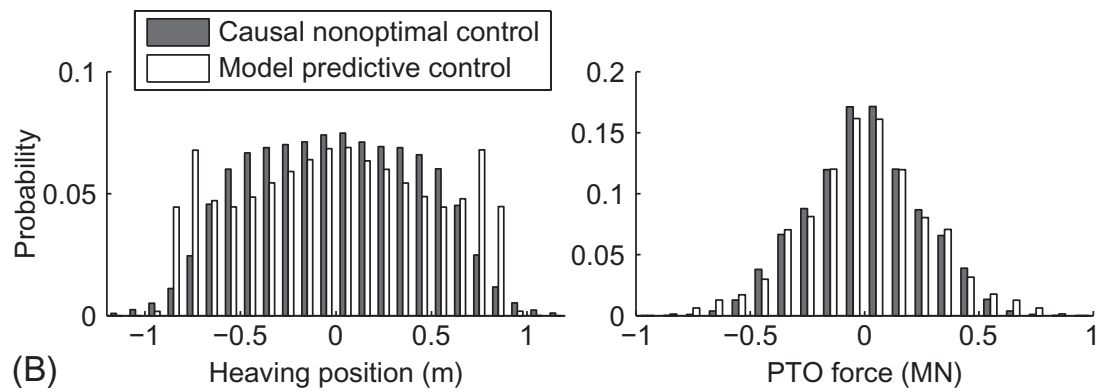

FIG. 12.5 Performance of proposed nonoptimal causal control, compared with optimal MPC. Relative capture width figures of merit are comparable for both controllers, with some small compromise on PTO force limits by the SE controller. (A) Relative capture width (RCW). (B) Distribution of heaving excursion and PTO force. 
fraction of the computational complexity of MPC. Note also, from Fig. 12.5, that the amplitude and force limits of $\pm 1 \mathrm{~m}$ and $\pm 1 \mathrm{MN}$ are, in general, well respected. The parameter $\lambda$ in Fig. 12.5 reflects the sea spectrum bandwidth $(\lambda=0.5 \Rightarrow$ widebanded), from the Ochi sea spectrum model (Ochi, 1998). Further details and results for this controller are given in Fusco and Ringwood (2013).

\subsubsection{The 'Aalborg' PID Controller}

The control algorithm reported here follows a control signal parameterization which is a direct consequence of the WEC model employed. For a WEC model based on the typical Cummins formulation (see Chapter 3 and Cummins, 1962), the control force is parameterized (Nielsen et al., 2013) as:

$$
f_{c}(t)=m_{c} \dot{v}(t)+c_{c} v(t)+k_{c} u(t),
$$

where $v(t)$ and $u(t)$ are the device velocity and displacement, respectively. It can reasonably be argued that this controller parameterization is optimal for monochromatic waves, since it effective has the potential to cancel or modify each of the terms in the Cummins' equation model, bearing in mind that the radiation damping term is constant for a fixed frequency (the monochromatic case). There is also some familiarity to the form of Eq. (12.14) since, from a velocity perspective, the control signal is composed of proportional $\left(c_{c} v(t)\right)$, integral $\left(k_{c} u(t)\right)$, and derivative $\left(m_{c} \dot{v}(t)\right)$ terms, following the pattern of the well-known PID form used in traditional feedback control. However, one important distinction with the traditional PID controller is that the controller in Eq. (12.14) is a feedforward controller and does not operate on a tracking error, as does the traditional PID controller. Nevertheless, there is a bidirectional interaction between the control force, $f_{c}(t)$, and the device velocity, $v(t)$, via the WEC system or system model $\left(f_{c}(t) \rightarrow v(t)\right)$ and via the control calculation $\left(v(t) \rightarrow f_{c}(t)\right)$.
For the panchromatic case, the radiation damping term in the WEC model is replaced by a finite-order rational approximation (see Chapter 3) and the (now) stochastic optimal control problem is solved using a variational approach based on a Hamiltonian formulation. The resulting control force solution is:

$$
\begin{aligned}
f_{c}(t)= & -\left(m+m_{\infty}\right) \dot{v}(t)-k_{b} u(t) \\
& +\int_{t}^{\infty} h_{r v}(\tau-t) v(t) d \tau,
\end{aligned}
$$

where $m$ is the WEC inertia, $m_{\infty}$ is the infinite frequency added mass, $k_{b}(t)$ is the restoring force constant (assuming a linear restoring force), and $h_{r v}(t)$ is the impulse response resulting from the rational approximation to the radiation damping kernel. It can be noted that Eq. (12.15) maintains the general PID form, with the proportional term now replaced with a convolution representing the radiation damping term. Effectively, the terms in Eq. (12.15) cancel each term in the Cummins equation, resulting in perfectly optimal control (within the bounds of the approximation of the radiation damping kernel), assuming that the Cummins equation model is exact.

Since the integral term in Eq. (12.15) requires future knowledge of $v(t)$, the authors further develop a causal version of the controller, which requires no future knowledge. The interested reader is referred to Nielsen et al. (2013) for the complete details.

\subsubsection{WEC Controllers Based on Numerical Optimization}

The class of WEC controllers based on numerical optimization share the following characteristics:

- An accurate model of the WEC is required,

- The complexity of the controller is such that it exploits all the subtleties of the model, ie, the controller is highly tuned to the model,

- No fixed parameterization is applied to the control force (as, for example, in Sections 
12.2.3.1 and 12.2.3.2, but rather a solution for the control signal time series is sought from numerical optimization,

- Device constraints (displacement, force, etc.) can be included in the control formulation, and

- The computational demands of such controllers are significant, mainly due to the iterative numerical optimization.

This class of controller has emerged from traditional feedback control and, specifically, MPC, which was developed for the relatively slow process and chemical industry. In the WEC setting, the traditional feedback error cost function is replaced with a performance function of the form:

$$
J(T)=\int_{0}^{T} f_{p t o}(t) v(t) d t,
$$

where $T$ is the control horizon, $f_{p t o}(t)$ the desired optimal PTO force (the solution of the optimization problem) and $v(t)$ the device velocity. A variety of MPC formulations have been reported in the literature, including Hals et al. (2011b), Cretel et al. (2011), Brekken (2011), and Li and Belmont (2014). The main differences between these formulations are the specific cost functions employed, which are usually variations on Eq. (12.16) designed to ensure a convex optimization problem, which simplifies the calculation of the optimum. A recent variation on the general MPC framework has been developed (Bacelli and Ringwood, 2015), inspired by the emergence of pseudo-spectral optimal control formulations within the traditional control community, which parameterizes the WEC signals with basis functions, resulting in a computationally efficient formulation. An outline of the solution route is provided here.

This control solution is based on the discretization, in the time domain, of the PTO force and of the motion of the device in order to transform the problem into a nonlinear program (NLP). The approach is similar to the direct simultaneous method used for the solution of optimal control problems (Cuthrell and Biegler, 1987), where both the control variables and the state variables are discretized. The optimal control problem is to find the PTO force vector $f_{p t o}$ that maximizes the total absorbed energy $J$, subject to the equation of motion (Cummins equation) and the additional control and motion path constraints described as:

$$
\begin{gathered}
\boldsymbol{h}\left(u, v, f_{p t o^{\prime}} t\right)=0 \\
\boldsymbol{g}\left(u, v, f_{p t o}, t\right) \leq 0 .
\end{gathered}
$$

where $u(t)$ is the device displacement. The discretization of the control problem is performed by approximating the position $(u)$ and the velocity $(v)$ vectors with a linear combination of the basis functions $\phi_{k}(t)$, and the PTO force $\left(f_{p t o}\right)$ vector with a linear combination of the functions $\phi_{k}^{P}(t)$; the $i$ th components of these vectors are:

$u_{i}(t) \approx u_{i}^{N}(t)=\sum_{k=1}^{N} x_{i k}^{u} \phi_{k}(t)=\Phi(t) \hat{x}_{i}^{u}, \quad i=1, \ldots, n$

$v_{i}(t) \approx v_{i}^{N}(t)=\sum_{k=1}^{N} x_{i k}^{v} \phi_{k}(t)=\Phi(t) \hat{x}_{i}^{v}, \quad i=1, \ldots, n$

$$
\begin{aligned}
& f_{p t o_{i}}(t) \approx f_{p t o_{i}}^{N^{P}}(t)=\sum_{k=1}^{N^{P}} u_{i k} \phi_{k}^{P}(t)=\Phi^{P}(t) \hat{\boldsymbol{u}}_{i}, \\
& \quad i=1, \ldots, m,
\end{aligned}
$$

where $N$ and $N^{P}$ are the orders of the expansions for the states (position and velocity) and the control input (PTO force), respectively,

$$
\begin{aligned}
& \hat{\boldsymbol{x}}_{i}^{u}=\left[\hat{x}_{i 1}^{u}, \hat{x}_{i 2}^{u}, \ldots, \hat{x}_{i N}^{u}\right]^{\mathrm{T}}, \hat{\boldsymbol{x}}_{i}^{v}=\left[\hat{x}_{i 1}^{v}, \hat{x}_{i 2}^{v}, \ldots, \hat{x}_{i N}^{v}\right]^{\mathrm{T}}, \\
& \hat{\boldsymbol{u}}_{i}=\left[\hat{u}_{i 1}, \hat{u}_{i 2}, \ldots, \hat{u}_{i N_{P}}\right]^{\mathrm{T}},
\end{aligned}
$$

and

$$
\begin{aligned}
\Phi(t) & =\left[\phi_{1}(t), \phi_{2}(t), \ldots, \phi_{N}(t)\right], \\
\Phi^{P}(t) & =\left[\phi_{1}^{P}(t), \phi_{2}^{P}(t), \ldots, \phi_{N_{P}}^{P}(t)\right] .
\end{aligned}
$$


Using the approximated velocity and PTO force, from Eqs (12.20) and (12.21), respectively, the total absorbed energy $J^{N}$ is

$$
J^{N}=-\int_{0}^{T} \boldsymbol{\Phi}(t) \boldsymbol{X}^{v} \boldsymbol{U}^{\mathrm{T}} \boldsymbol{\Phi}^{P^{\mathrm{T}}}(t) d t=\sum_{i=1}^{N^{P}} \sum_{j=1}^{N} w_{i j} \Gamma_{i j}
$$

where $w_{i j}$ are the elements of the matrix $\boldsymbol{W}=\boldsymbol{X}^{v}$ $U^{\mathrm{T}}$, which depends on the coefficients of the velocity $\left(\boldsymbol{X}^{v}\right)$ and the PTO force $(\boldsymbol{U})$, while $\Gamma_{i j}$ are the elements of the constant matrix $\Gamma$ which depends on the bases $\Phi$ and $\Phi^{P}$ as

$$
\Gamma=\int_{0}^{T} \Phi^{\mathrm{T}}(t) \Phi^{P}(t) d t
$$

The matrices $\boldsymbol{X}^{u}, \boldsymbol{X}^{v}$, and $\boldsymbol{U}$ are defined as

$$
\begin{aligned}
\boldsymbol{X}^{u} & =\left[\hat{\boldsymbol{x}}_{1}^{u}, \ldots, \hat{\boldsymbol{x}}_{N}^{u}\right], \quad \boldsymbol{X}^{v}=\left[\hat{\boldsymbol{x}}_{1}^{v}, \ldots, \hat{\boldsymbol{x}}_{N}^{v}\right], \\
\boldsymbol{U} & =\left[\hat{\boldsymbol{u}}_{1}, \ldots, \hat{\boldsymbol{u}}_{N_{P}}\right] .
\end{aligned}
$$

The result of the discretization is the finite dimensional NLP described by the quadratic cost function $J^{N}$ in Eq. (12.22), the linear equality constraints due to the dynamic equations (Cummins equation) which is reformulated in terms of the variables in Eqs (12.19)-(12.21), and by the additional equality and inequality path and control constraints in Eqs (12.17) and (12.18).

The solution to this discretized WEC control problem is provided by the optimal PTO force, $\hat{\boldsymbol{u}}^{*}$, corresponding to the discrete parametric form of the PTO force in Eq. (12.21). For the unconstrained case, an algebraic solution results while, with the addition of constraints, a numerical optimization is required. For the full development and solution, the interested reader is referred to Bacelli and Ringwood (2015). One useful feature of the pseudo-spectral approach is that a user-defined trade-off between computational complexity and fidelity of approximation can be achieved by appropriate choice of $N$ and $N^{P}$ in Eqs (12.19)-(12.21), which guarantees a feasible real-time control solution. In the discretization in Eqs (12.19)-(12.21), a variety of basis function types can be used; however, a natural choice of basis function is a Fourier series, given the harmonic nature of the system variables and the excitation signal.

\subsubsection{Control of WEC Arrays}

The analytical formulation of the maximum power absorbed by an array of oscillating devices was independently derived by Evans (1979) and Falnes (1980). Both authors obtained a result which is the general case of reactive (phase and amplitude control) control. A system of optimally controlled WECs is described by Evans (1981), with the effect of motion constraints on maximum power absorption also considered. Also, Falnes and Budal (1982) reported a study on linear arrays of heaving buoys, where they considered unconstrained motion as well as constrained motion. Falnes extends his previous work in Falnes (1984), where he considers an infinite linear array of evenly spaced oscillating bodies. Constraints on arrays of oscillating bodies have also been considered in Fitzgerald and Thomas (2007), where limiting the oscillation amplitude to two or three times the incoming wave amplitude results in the positive interference between array elements being reduced, though the negative interference is not significantly affected.

Finally, a real-time control algorithm for arrays of WECs, using a basis function parameterization of system variables (following the general development in Section 12.2.3.3), is presented in Bacelli et al. (2013), while the method is extended to consider system constraints in Bacelli and Ringwood (2013a). All of these methods use a Cummins equation-style model, with the device variables now expanding to vectors to cater for the multiple bodies (see Chapter 8). Though coordinated control of WEC arrays has been shown to be advantageous (up to 20\% more energy can be captured by getting the devices to work together and take 
advantage of mutual interaction through radiation), there is a significant dependence on the model accuracy and an appreciable reduction in coordinated control benefit can accrue from modelling errors. To this end, a sensitivity analysis to device position errors is carried out in Bacelli et al. (2013), which shows that independent device control is more beneficial than coordinated control, when modelling errors (in relative device position) exceed a certain threshold.

\subsubsection{Wave Forecasting}

While some WEC control algorithms circumvent the need to predict future variations in free surface elevation or excitation force (Fusco and Ringwood, 2013; Scruggs et al., 2013), in general there is a need to provide forecast values of free surface elevation or excitation force due to the noncausality of the optimal PTO force, as articulated in Section 12.2.2. Fortunately, there is a strong positive connection between the wave forecasting requirements of energy maximizing control (Fusco and Ringwood, 2012) and the forecastability of random seas (Fusco and Ringwood, 2010), due to the close relationship between the radiation damping dynamics and the design sea state (ie, predominant period).

Wave forecasting can be performed using upwave measurement, based on either black-box propagation models (Price and Wallace, 2007; Eder et al., 2013; Monk et al., 2013) or models for wave propagation based on wave physics (see Chapter 10). A simpler alternative is to use a time series model at the device location (Fusco and Ringwood, 2010) for either excitation force or free-surface elevation. Both approaches are illustrated in Fig. 12.6. A comparative case study (Paparella et al., 2015) of both up-wave and timer series approaches showed little advantage of the inclusion of up-wave measurements, suggesting that the additional cost of upwave sensors and the development of (either time-series or physics-based) wave propagation models is questionable. While many time series techniques may be employed, including harmonic, neural network, and models based on the EKF, a simple linear autoregressive (AR) forecasting model, viz:
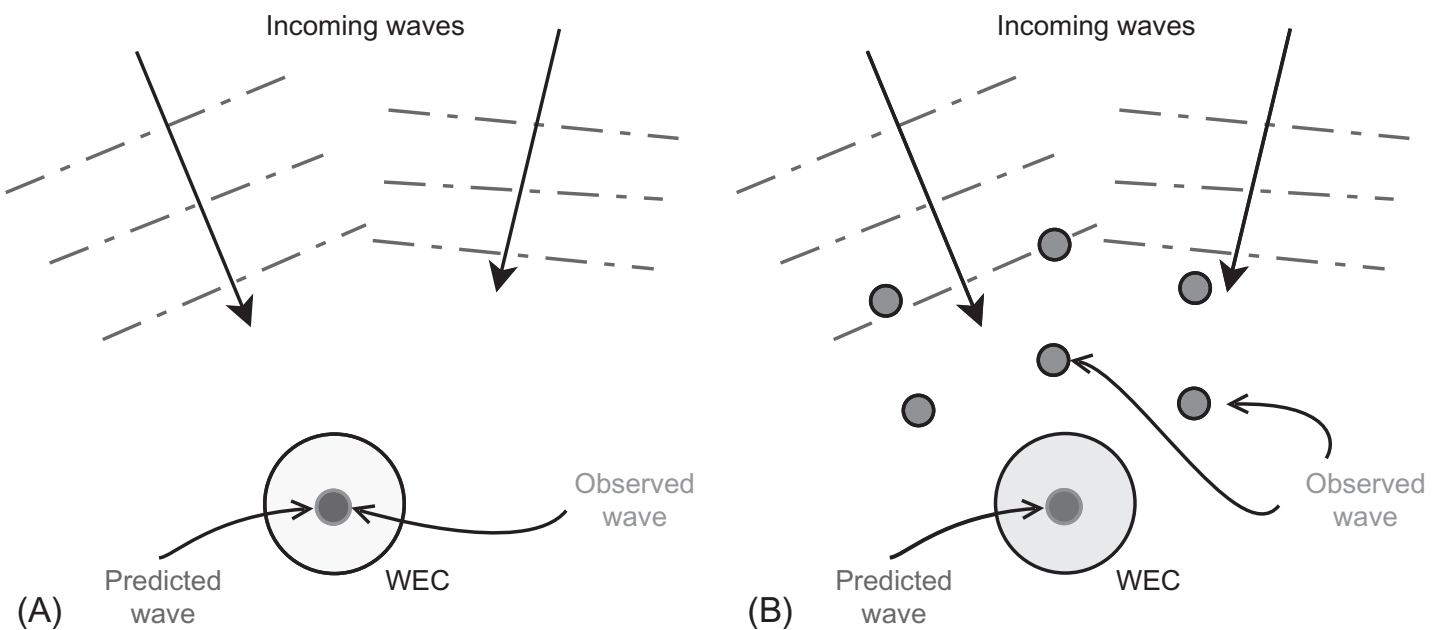

(A)

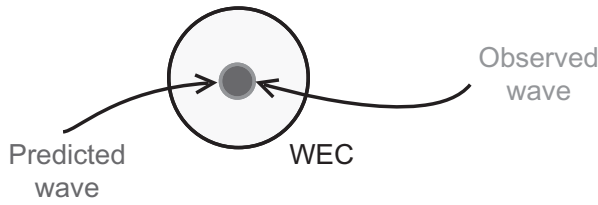

(B)

FIG. 12.6 The two main approaches to wave forecasting. Up-wave prediction requires the addition of extra sensors, while the time series approach in (A) simply forecasts future excitation force based on the measured device motion. (A) Prediction based only on local single-point measurements. (B) Prediction based on up-wave measurements. 


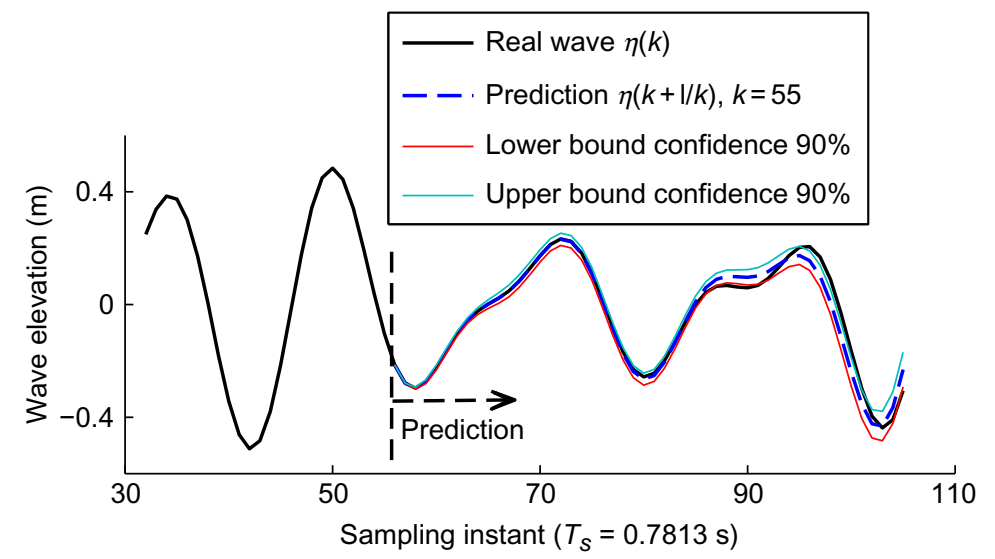

FIG. 12.7 Wave prediction and confidence intervals for a filtered wave record corresponding to Pico Island in the Azores. The prediction shows acceptable fidelity over a $35 \mathrm{~s}$ forecast horizon.

$$
\hat{\eta}(k+l \mid k)=\sum_{i=1}^{n} \hat{a}_{i}(k) \hat{\eta}(k+l-i \mid k),
$$

performs well and has a nice frequency domain interpretation. For example, Fig. 12.7 shows $\hat{\eta}(k+l \mid k)$, for $l=1$ to $l=50$, at a specific time instant $k$, calculated with an AR model of order $n=24$, for wave data at Pico Island in the Azores filtered with cut-off frequency $\omega_{c}=0.7 \mathrm{rad} / \mathrm{s}$.
Fig. 12.8 shows how the AR model poles pick out the characteristic spectral peaks in the sea spectrum.

\subsubsection{WEC Control Perspectives}

A number of comments, from a control perspective, may reasonably be made in relation to the WEC models (and array models) upon
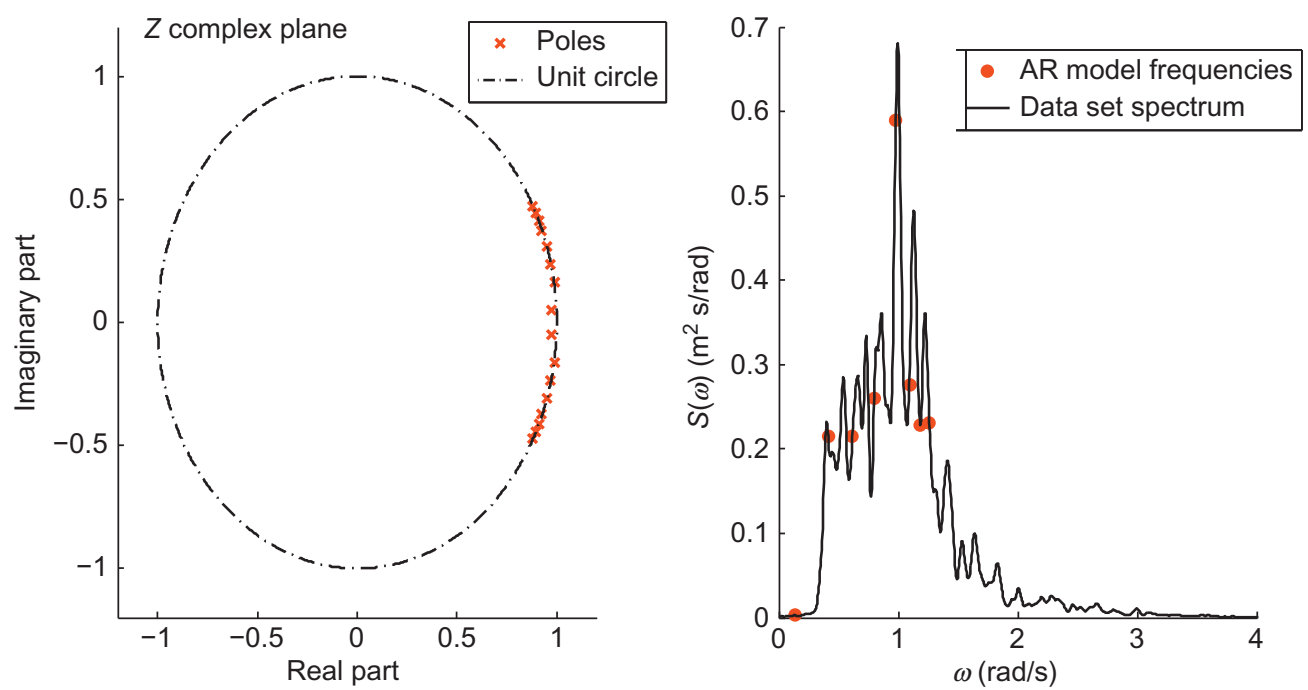

FIG. 12.8 AR model poles and corresponding sea spectrum. The AR model is essentially a time domain model; the pole locations give a useful frequency-domain interpretation, which can be well related to the sea spectrum. 
which WEC controllers are based. In the first instance, the robustness of WEC control systems to modelling errors is highlighted in Fig. 12.1, which shows the calculation of the optimal velocity profile as an open loop calculation, which is therefore sensitive to modelling errors. There is therefore a robustness issue, which is rarely explicitly addressed in WEC control studies, though there are some exceptions, for example, Fusco and Ringwood (2014).

In general, the more complex the WEC model, the more highly tuned the model-based controller will be. While such complex controllers potentially offer better performance, they are likely to be more sensitive to modelling error. The control formulation in Sections 12.2.3.112.2.3.3 offer incrementally increasing complexity, but some consideration needs to be given to the robustness to modelling error. To this end, the pseudo-spectral formulation shown in Section 12.2.3.3 could offer a variable sensitivity to modelling errors, through the appropriate choice of $N$ and $N^{P}$, though this requires further study. One interesting experiment was reported in Kracht (2013), where the performance of the simple controller reported in Section 12.2.3.1 was compared to an MPC in a tank test for a heaving buoy device. Though the MPC (marginally) outperformed the simple controller in simulation (where the same model was used in simulation as for the model-based controller) (Fusco and Ringwood, 2013), the simple controller was seen to outperform the MPC in the tank test (Kracht, 2013), most likely due to the fact that the model-based MPC was more sensitive to modelling errors.

While the bulk of WEC control design methods are based on linear WEC models, nonlinear WEC modelling methods are becoming more common (see Chapters 5-7 and Retes et al., 2015) and WEC controllers based on nonlinear WEC models are also beginning to appear. One important issue raised in Retes et al. (2015) is that, while (linear) WEC models may validate well with tank tests for uncontrolled systems, the operational range of the
WEC may be significantly expanded under control, with greater extremes in wetted surface variation (displacement) and velocity/acceleration likely to be achieved. In such an expanded operational situation, nonlinear models based on boundary-element methods (see Chapter 5 and Retes et al., 2015), or nonlinear or representative linear models identified from wave tank data (see Chapter 7) may give greater fidelity. However, apart from the representative linear models (Davidson et al., 2015), WEC controllers have yet to be designed for WEC models which include nonlinear Froude-Krylov forces. However, controllers are available for WEC models containing:

- Nonlinear restoring force (Richter et al., 2013; Fusco and Ringwood, 2014),

- Nonlinear viscous damping (Bacelli and Ringwood, 2014), and

- Nonideal PTO efficiency characteristics (Falcão et al., 2015; Bacelli et al., 2015; Genest et al., 2014).

This chapter presents a small selection of WEC control algorithms and perspectives. A broader study on the WEC control systems problem and literature is available in Ringwood et al. (2014). In general, there is a positive correlation between the complexity of the model that a WEC controller is based on and the potential power capture performance. However, increased model complexity is also accompanied by an increased computational burden and, most importantly, a WEC controller tightly tuned to a complex WEC model may be quite sensitive to modelling errors. With the almost exclusive reliance on linear WEC models for WEC model-based controllers, and the dubious validity of linear WEC models under control conditions (Retes et al., 2015), significant care needs to be taken that an overall well-performing WEC control solution is achieved. While traditional (feedback regulatory) controllers are often reasonably based on linearized system models, since system operation is forced to an equilibrium by the controller action, WEC 
controllers tend to exaggerate motion away from equilibrium (eg, the still water level) making the assumption of small variations invalid.

\subsection{OPTIMIZATION OF WECs AND WEC ARRAYS}

This section considers the use of numerical optimization to optimize the geometry of WECs and the layout of WEC arrays. In particular, the interaction between such device/array optimization and the control design problem is also studied and some recommendations made. In theory, all the design parameters of single-body WECs, multibody WECs and WEC arrays can be numerically optimized, providing there exists:

(a) A suitable objective function to provide a figure of merit for the various candidate designs, and

(b) A route to calculate the objective function values

Regarding (a), a typical figure of merit employed is the amount of useful energy converted by a WEC or WEC array. However, focussing on power alone may result in disproportionate capital or other costs and a more holistic objective is suggested by the techno-economic optimization framework outlined in Costello et al. (2012) and illustrated in Fig. 12.9. In the techno-economic optimization framework, significant computation is involved, including the required recalculation of hydrodynamic parameters in the 'device analysis' block, as the WEC geometry is adapted. However, providing such a problem can be solved, the resulting solution balances capital, operation and other costs against power production, maximizing the economic benefit of a wave energy project. With regard to (b), while the specification of suitable WEC models for such a formulation is not too difficult (and is the focus of much of this book), the accurate articulation of the cost model is far from trivial, as outlined in Teillant et al. (2012).

Finally, there are (in general) no guarantees that the global minimum (or maximum) of the objective function can be found in practice. For relatively simple performance functions, typically employing quadratic measures, the optimization problem presented is usually convex, with a guaranteed convergence to the function minimum or maximum. However, for more complex objective functions (and more complex relationships between the objective function variables, ie, the device model), convergence is not guaranteed, though recourse can be made to concurrent search algorithms (such as genetic algorithms Goldberg, 1989), which employ multiple interacting simultaneous solutions to cover the search space.

In the following two sections, by way of example, two optimization problems of interest to WEC developers are presented. In Section 12.3.1 the geometric optimization of a heaving buoy is

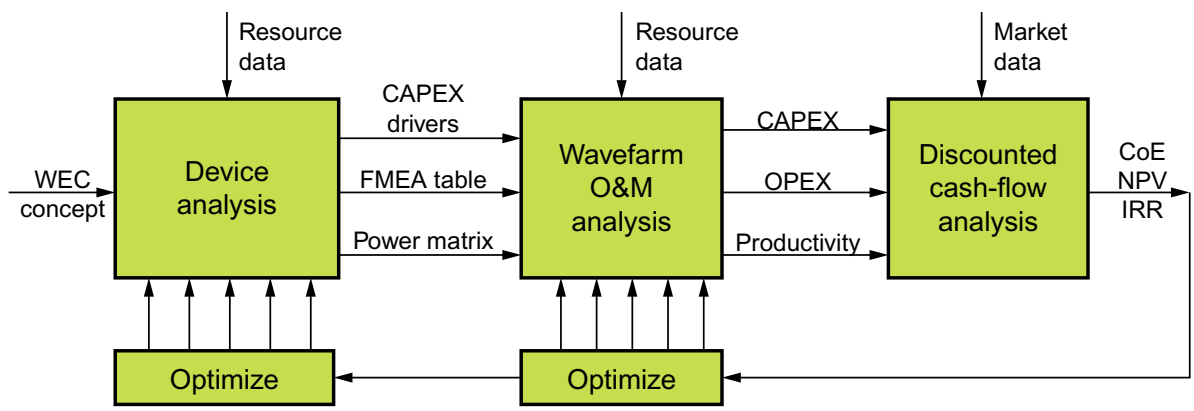

FIG. 12.9 Techno-economic optimization philosophy. 
considered, while in Section 12.3.2, the optimal spacing of an array of WECs in an array is considered. In both cases, the interaction between the geometric/spacing design and the control design problem is emphasized, highlighting the need to consider both design problems concurrently, rather than sequentially.

\subsubsection{Geometric Optimization of WECs}

Here, the optimization of geometric parameters of a WEC is considered. In reality, the impact of WEC geometry changes should be considered from many aspects of a wave energy project, as articulated in Fig. 12.9, including impacts on capital cost, transport costs, manufacturability, etc. However, for simplicity, the current analysis will focus exclusively on the maximization of energy capture, which will also facilitate a clear presentation of the interplay between geometry optimization and energy-maximizing control design. The overall combined geometry/control optimization is illustrated in Fig. 12.10. For each

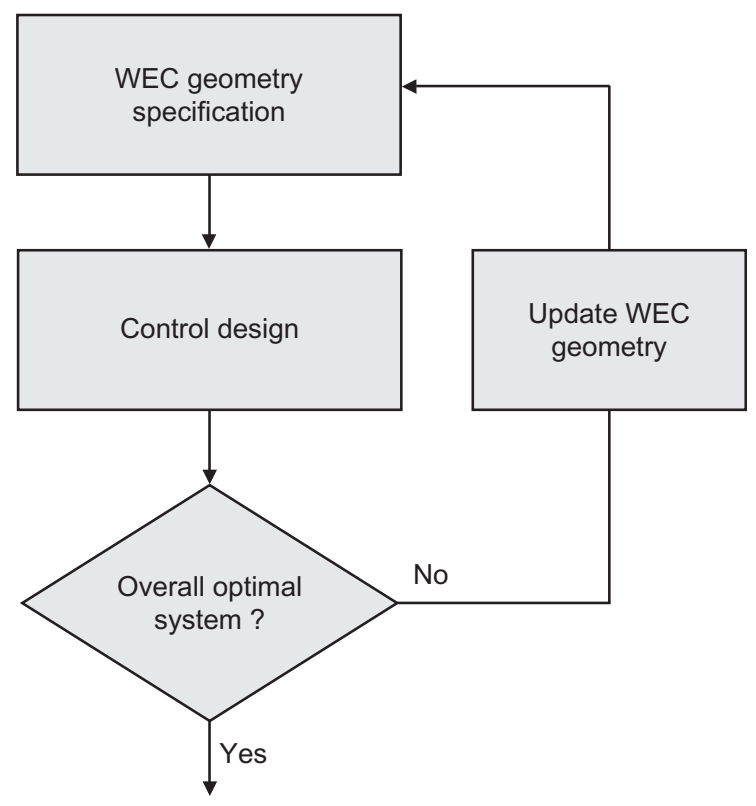

FIG. 12.10 Combined geometric and control optimization. trial of the WEC geometry parameters, the control system must be redesigned to ensure that energy capture is maximized over the full range of operation.

In general, the WEC geometry is designed for the prevailing sea conditions in a particular location, with control subsequently used to improve the energy conversion performance of the device for sea-states other than the design sea-state. This usually involves matching the device frequency response, or response amplitude operator (RAO), to the predominant sea spectrum. However, particular control strategies, subsequently employed and while doing their best to broaden the RAO in frequency range, can be limited in capability to produce an overall optimal system. Two cases in point highlight the issue. Latching control (Budal and Falnes, 1975; Babarit and Clément, 2006a), which delays the motion of a WEC by locking its position at the extreme (upper and lower) points of motion, has the ability to slow down the WEC in sea periods longer than the design period. On the other hand, declutching control (Babarit et al., 2009; Folley and Whittaker, 2009) has the opposite effect-for intervals of the motion cycle, the WEC is 'unloaded' allowing the response to be speeded up in sea periods shorter than the design period. Both latching and declutching produce composite device/control ('closed loop') systems, which are nonsymmetrical with respect to the predominant sea state.

Aiming to improve wave energy extraction, a number of studies have been done on device geometry optimization of WECs independently of the control system (Babarit and Clément, 2006b; Alves et al., 2007; McCabe et al., 2010; McCabe, 2013; Kurniawan and Moan, 2013). However, a preliminary study, examining the influence of latching control on the overall geometric optimization of a heaving cylinder (Gilloteaux and Ringwood, 2010) suggested that significant interaction potentially existed between the geometry and control design problems, highlighting the issue for latching control. 
Some sample results, which illustrate the effect of variations in the radius, $r$, and draught, $d$, of a cylindrical point absorber WEC, are shown in Fig. 12.11 for four different control strategies:
UD Uncontrolled device (optimal passive damping, determined for peak for wave spectrum),

LD Latching-controlled device (Babarit and Clément, 2006a),

(A)
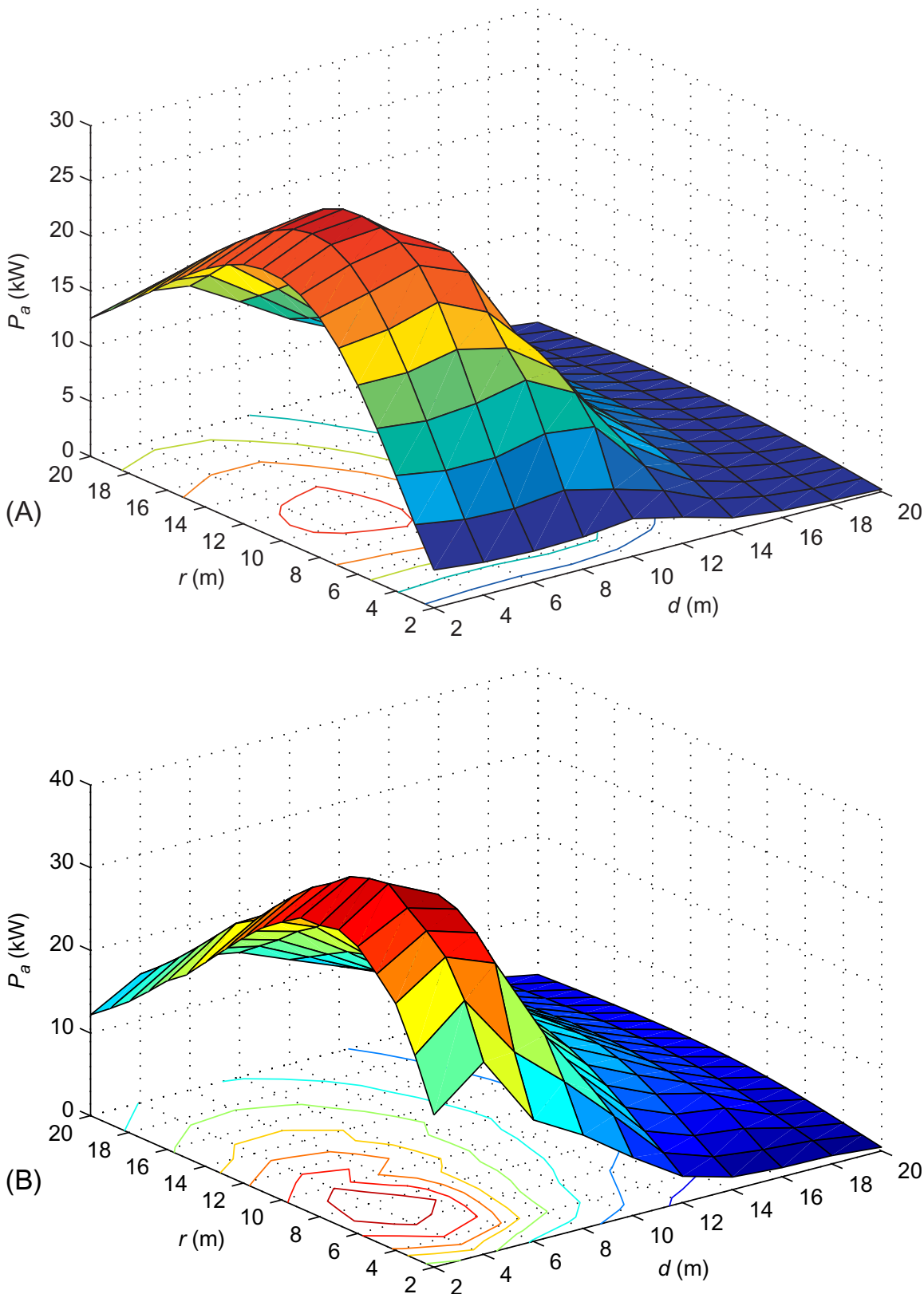

FIG. 12.11 (Continued) 

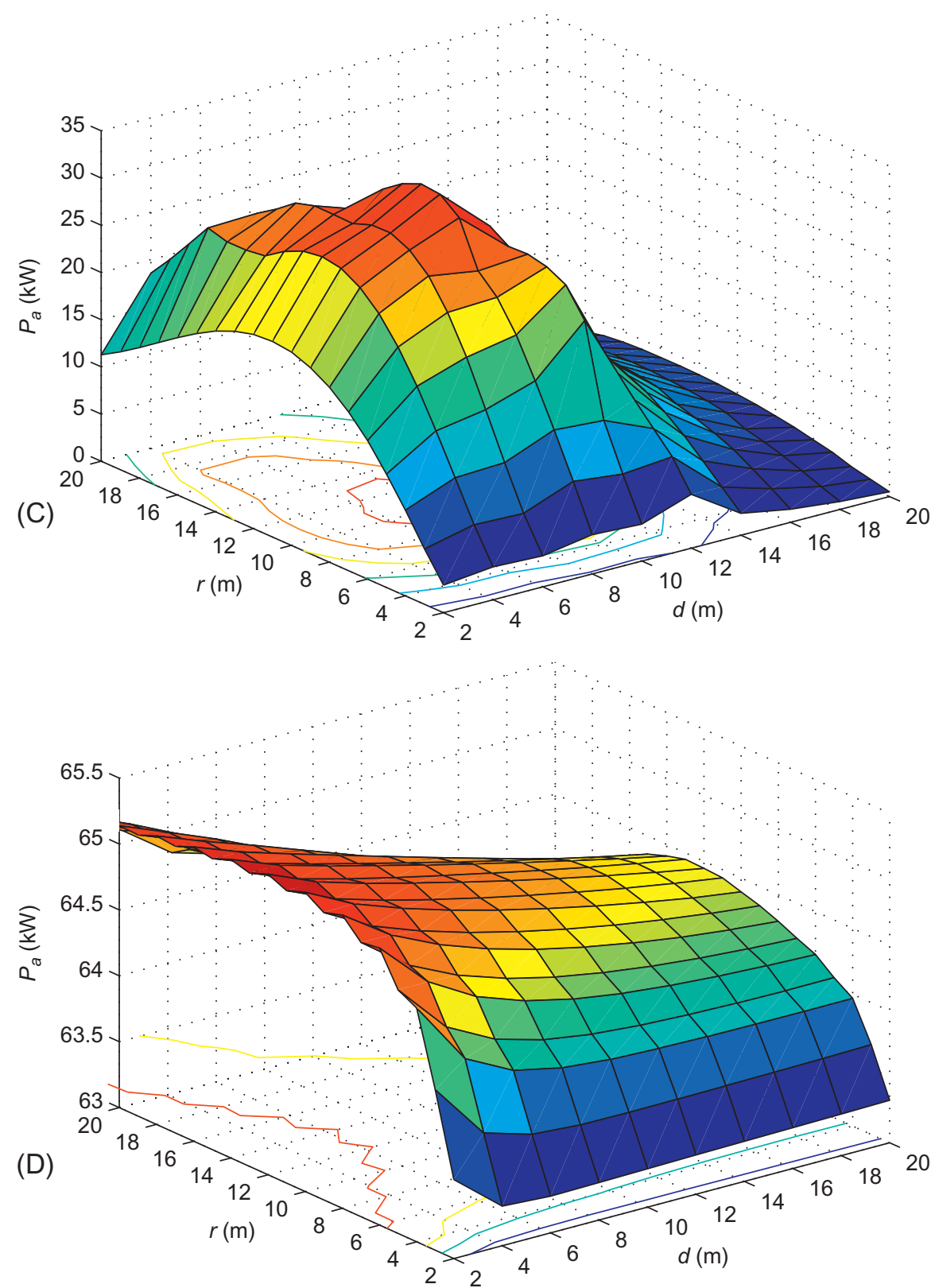

FIG. 12.11 Evolution of the absorbed power for different values of $r$ and $d$ : (A) UD, (B) LD, (C) DD, (D) MPCD (polychromatic waves: $H_{s}=1 \mathrm{~m} ; T_{p}=7 \mathrm{~s}$ ). 
DD Declutching-controlled device (Babarit et al., 2009), and

MPCD MPC-like controlled device (Bacelli and Ringwood, 2015).

$P_{a}$ is the absorbed power and the contour plots on the base of the surface plots clearly show the diversity in the optimal values for $r$ and $d$ (denoted $r^{*}$ and $d^{*}$ respectively, which are enumerated in Table 12.1. $T_{r}^{*}$ is the resonant period of each of the optimized devices. Note that $P_{a} / V_{o}$ is calculated for information only, since the performance objective is the maximization of $P_{a}$. If desired, an alternative performance function, with a dependence on (for example) $P_{a} / V_{o}$, could be employed, which would yield different optimal geometries.

Significantly, since latching (LD) has the ability to slow down the motion of a WEC but not speed it up, the optimal WEC has a relatively small resonant period, while the converse is true for declutching (DD). The MPC-like strategy has the capability to both speed up and slow down the WEC, so the $T_{r}^{*}$ for the MPC-optimized device is fairly central and close to that for the UD case.

In the example shown, a simple device geometry is employed, with just two parameters to be optimized. For more complicated shapes, if shape optimization is to be performed, the geometry needs to be parameterized in terms of a finite number of parameters. For curves, etc., such parameterization can become more challenging, with the potential need to parameterize curved sections using basis functions, such as polynomials. However, as the number of parameters to be optimized grows, so too does the complexity and dimensionality of the performance surface to be searched, reducing the likelihood of achieving a global optimum and incurring significant computational requirements. One other issue to consider is that, while shapes (and controllers) may present advantageous average power capture performance, the displacement variations and maximum forces generated may also vary, and these also have cost implications, though the trade-off between force and amplitude constraints may also, to some extent (Bacelli and Ringwood, 2013b), be optimized. A more complete treatment of the interaction between geometry and control optimization is given in Garcia-Rosa and Ringwood (2015).

\subsubsection{WEC Array Layout Optimization}

Wave farms have a feature, unusual among the range of renewable energy technologies (such as solar, wind, and tidal), whereby the total energy output from a farm of $n$ devices can exceed that of $n$ individual devices. This phenomenon results from the fact that oscillating WECs radiate waves, which can constructively interfere with the incident wave field resulting in an overall net benefit to the devices in the array. However, by the same mechanism, destructive interference can also occur. Ultimately, array layouts and orientation need to be optimized for the prevailing conditions at the wave farm site.

TABLE 12.1 Optimal Geometries, Relative Size of the Various Optimized Devices and Relative Power Captured per Unit Volume

\begin{tabular}{llllrrr}
\hline Control & $r^{*}(\mathbf{m})$ & $d^{*}(\mathrm{~m})$ & $T_{r}^{*}$ & $V_{o}\left(\mathrm{~m}^{3}\right)$ & $\boldsymbol{P}_{a}(\mathrm{~W})$ & $\boldsymbol{P}_{a} / V_{o}\left(\mathrm{~W} / \mathrm{m}^{3}\right)$ \\
\hline UD & 11 & 6 & 7.06 & 2280.80 & 26,340 & 11.55 \\
LD & 6 & 4 & 5.55 & 452.39 & 39,730 & 87.82 \\
DD & 12 & 8 & 7.79 & 3619.11 & 33,290 & 9.20 \\
MPCD & 12 & 4 & 6.56 & 1809.56 & 65,130 & 35.99 \\
\hline
\end{tabular}


In addition, like the WEC geometry optimization discussed in Section 12.3.1, optimal wave farm layout is also a function of the control system employed in the farm. Both independent (each device has its own autonomous control system) and global (a coordinated control strategy, taking account of device interaction is employed) strategies result in different optimal farm layouts. The normal figure of merit for a wave farm is the $q$ factor, defined (Budal, 1977) as:

$$
q=\frac{W}{n W_{s}}
$$

where $W$ is the total energy absorbed by the array, $W_{s}$ is the energy absorbed for a single isolated device, and $n$ is the number of units in the array. If wave interactions have a constructive effect on the energy absorbed by the array, then $q>1$ and, if the effect is destructive, $q<1$.

A simple example (described more fully in Garcia-Rosa et al., 2015) is used to illustrate some of the issues. A small set of linear, triangular, and square arrays is examined and a single parameter (the interdevice spacing) is optimized, together with the incident wave orientation. It may be noted (Thomas and Evans, 1981) that the average $q$ factor around all possible wave directions (ie, array orientation) is unity; however, since most wave energy sites have a predominant wave direction, a favourable orientation (with $q>1$ ) can normally be found. For a square array of four cylindrical heaving WECs, with radius of $6.25 \mathrm{~m}$ and draught $4 \mathrm{~m}$, Fig. 12.12 shows the variation in $q$ factor for variations in array orientation and interdevice spacing (spacing distance $d$ is normalized by the device radius $r$ ). The upper and lower plots show the case for global array control (GC) (Bacelli et al., 2013) (using a control algorithm as described in Section 12.2.3.3) and passive tuning (optimal linear damping for each device, PC). A Bretschneider panchromatic wave spectrum with $H_{s}=1 \mathrm{~m}$ and $T_{p}=7 \mathrm{~s}$ was employed, using planar long-crested waves, and no PTO constraints were active.
A number of features of Fig. 12.12 are noteworthy. A distinct peak in the $q$ factor surface is evident, which implies a significant sensitivity to $d / r$ and $\beta$, suggesting that a poor choice for $d$ and $\beta$ can result in a very significant loss in energy conversion potential of the wave farm, at least for a particular, possibly the predominant, sea-state. It is also clear that significantly more energy can be converted using a coordinated control approach from the array ( $q$ factor peak is higher for global control case), as documented in Bacelli et al. (2013). In fact, with an optimized array layout and global control, up to $40 \%$ more power can be converted (Garcia-Rosa et al., 2015), compared to a layout-optimized array of devices with optimal damping. One other significant feature that can be drawn from Fig. 12.12 is that different optimal $d / r$ values are evident for the global control and optimal (passive) damping cases. Such a difference $\left(d / r^{*}=27\right.$ for GC and $d / r^{*}=23$ for PC, for the case illustrated in Fig. 12.12) shows the sensitivity of the optimal array layout to the control strategy employed. In general, there is little sensitivity of the optimal array orientation to the control strategy employed (Garcia-Rosa et al., 2015).

As an illustration of the significance of the sensitivity of the optimal array layout to the control strategy employed, Fig. 12.13 shows the corresponding optimal array layouts for linear ( 2 and 3 body), triangular, and square arrays for the GC and PC cases. The examples shown confirm that significant benefit can be obtained from optimizing the array layout and orientation and that the control strategy should also be considered at the array layout optimization stage. However, the simple examples shown in this section (chosen for clarity of illustration) employ only one free layout dimension parameter (and an orientation parameter) while, in practice, much more freedom could be employed. For example, a commercial array of (say) 30 devices might be parameterized in terms of the individual 

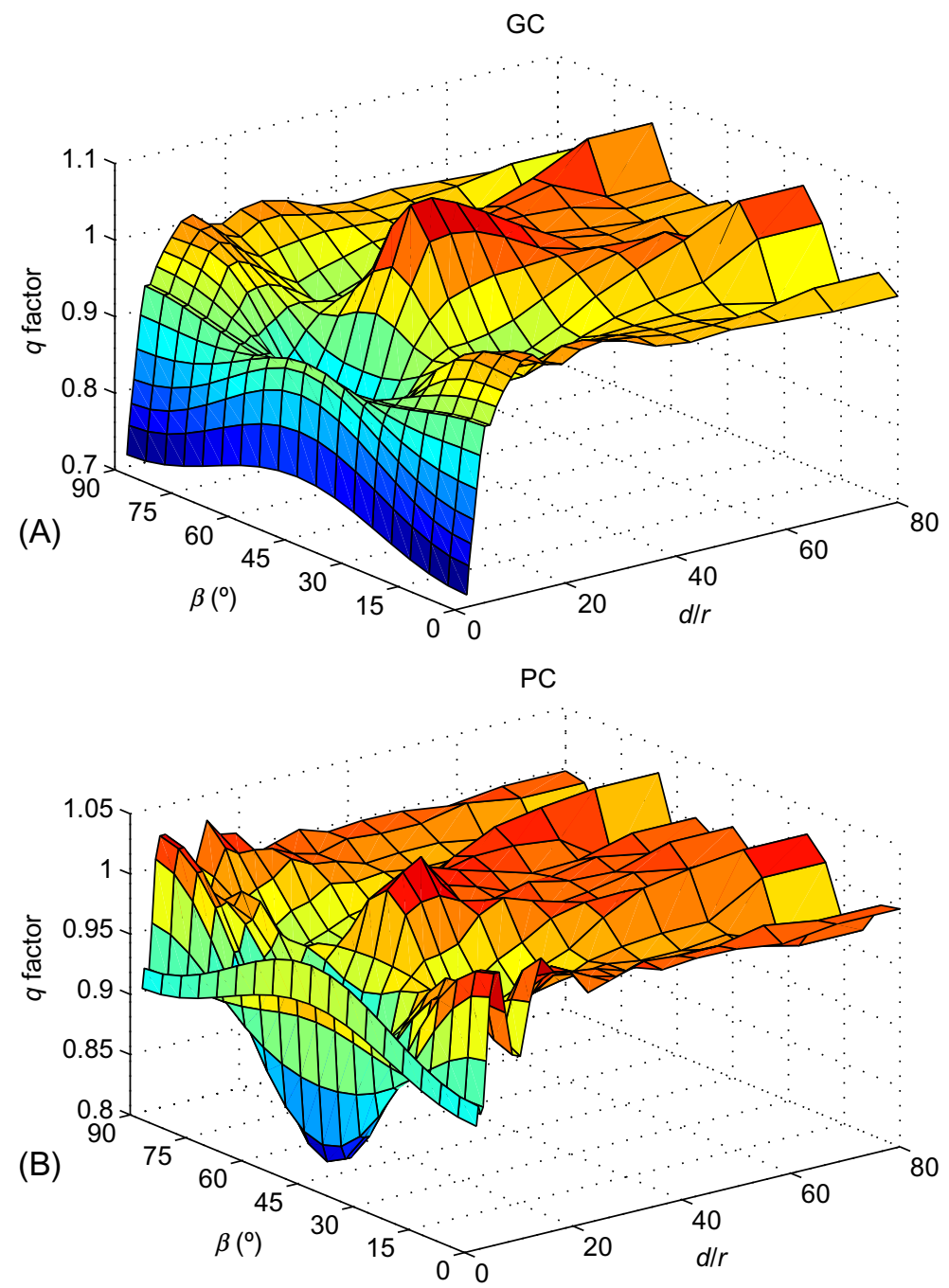

FIG. 12.12 L4, G2—q factor values for different $d / r$ and $\beta$ corresponding to (A) global control, and (B) passive optimal damping.

Cartesian $(x, y)$ coordinates, giving 60 parameters to be optimized. There are no guarantees that the resulting multidimensional optimization problem is convex, potentially requiring the employment of concurrent search algorithms, with the consequent high computational overhead. However, array layout design is a once-off design issue and, given the cost of WEC arrays and the potential impact on energy receipts of a suboptimal layout, deserves considerable attention. However, it must also be recognized that the final optimal array layout will also need to respect other design criteria relating to moorings, electrical interconnection, and navigational constraints. In addition, optimization across the full range of sea-states, and their associated probability, would need to be performed. 
L1

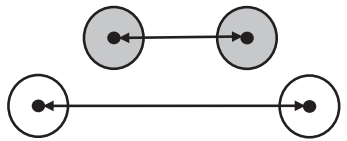

L2
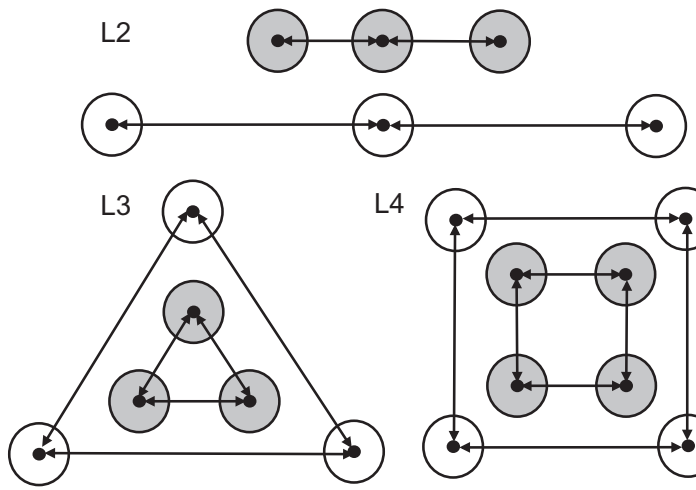

L4

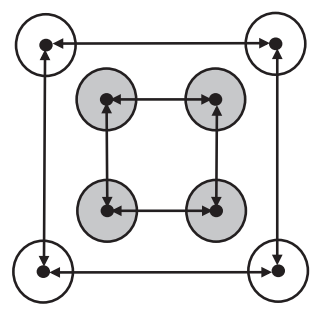

FIG. 12.13 Plan view of optimal array configurations for passive control (shaded) and global control (unshaded): L1 (scale $1: 100$ ), L2 (scale $1: 100$ ), L3 (scale $1: 200$ ), and L4 (scale $1: 400)$.

\subsubsection{Summary}

- For model-based control design, and the simulation of WEC controller performance, time-domain WEC models are required.

- Ideally, WEC controllers should respect the true nonlinear dynamics of WECs (both PTO and hydrodynamics); however, controllers based on linear WEC models predominate so far, due to the difficulty of generating true nonlinear controllers. This is due to the difficulty of nonlinear WEC controller design.

- There is, in general, a positive correlation between the complexity of the hydrodynamic models employed and the complexity of the resulting controllers. For real-time implementation, controller computational complexity can become an issue.

- The robustness of WEC controllers to modelling error should be considered in identifying an overall optimal controller, particularly since WEC controllers drive the system away from equilibrium and subject the WECs to forces that create relative body/fluid motion well in excess of that experienced due to wave motion alone. In addition, higher device/fluid relative velocities may be attained under control, resulting in the magnification of viscous drag effects.

- Optimal control is noncausal, but wave forecasting can assist in the provision of future variations in excitation force or free-surface elevation, and can be effectively achieved using autoregressive forecasting models.

- Optimization of WEC geometry and array layout should include consideration of the array control strategy as well as the incident wave climate.

- The significant amount of computation required for parametric (layout and geometry) optimization means that the WEC numerical model employed must be computationally efficient. This is especially true in the case where total wave farm project optimization is considered, as illustrated in Fig. 12.9.

\section{References}

Alves, M., Traylor, H., Sarmento, A., 2007. Hydrodynamic optimization of a wave energy converter using a heave motion buoy. In: Proc. of the 6th Int. Conf. on Wave and Tidal Energy. Porto, Portugal.

Babarit, A., Clément, A., 2006. Optimal latching control of a wave energy device in regular and irregular waves. Appl. Ocean Res. 28, 77-91.

Babarit, A., Clément, A., 2006. Shape optimisation of the searev wave energy converter. In: Proc. of the IX World Renewable Energy Congress. Florence, Italy.

Babarit, A., Guglielmi, M., Clément, A.H., 2009. Declutching control of a wave energy converter. Ocean Eng. 36 (12-13), 1015-1024.

Bacelli, G., Ringwood, J., 2013. Constrained control of arrays of wave energy devices. In: Proc. 11th European Wave Energy Conference. Aalborg, Denmark.

Bacelli, G., Ringwood, J., 2013. A geometric tool for the analysis of position and force constraints in wave energy converters. Ocean Eng. 65, 10-18.

Bacelli, G., Ringwood, J., 2014. Nonlinear optimal wave energy converter control with application to a flap-type device. In: Proc. 19th IFAC World Congress. pp. 7696-7701. 
Bacelli, G., Ringwood, J., 2015. Numerical optimal control of wave energy converters. IEEE Trans. Sustainable Energy 6, 294-302.

Bacelli, G., Genest, R., Ringwood, J.V., 2015. Nonlinear control of a flap-type wave energy converter with a non-ideal power take-off system. IFAC Annu. Rev. Contr. 40, 116126.

Bacelli, G., Balitsky, P., Ringwood, J., 2013. Coordinated control of arrays of wave energy devices-benefits over independent control. IEEE Trans. Sustainable Energy 4, 1091-1099.

Beirão, P., Mendes, M.J.G.C., Valério, D., Sá Da Costa, J., 2007. Control of the Archimedes wave swing using neural networks. In: 7th European Wave and Tidal Energy Conference (EWTEC).

Brekken, T., 2011. On model predictive control for a point absorber wave energy converter. In: IEEE PowerTech, Trondheim. pp. 1-8.

Budal, K., 1977. Theory for absorption of wave power by a system of interacting bodies. J. Ship Res. 21 (4), 248-253.

Budal, K., Falnes, J., 1975. A resonant point absorber of ocean-wave power. Nature 256, 478-479.

Budal, K., Falnes, J., 1982. The Norwegian wave-power buoy project. In: The Second Intern. Symp. on Wave Energy Utillization. pp. 323-344.

Costello, R., Teillant, B., Weber, J., Ringwood, J., 2012. Techno-economic optimisation for wave energy converters. In: Proc. Intl. Conf. on Ocean Energy.

Cretel, J.A.M., Lightbody, G., Thomas, G.P., Lewis, A.W., 2011. Maximisation of energy capture by a wave-energy point absorber using model predictive control. In: IFAC World Congress.

Cummins, W., 1962. The impulse response function and ship motions. Schiffstechnik 9, 101-109.

Cuthrell, J.E., Biegler, L.T., 1987. On the optimization of differential-algebraic process systems. AIChE J. 33 (8), 1257-1270.

Davidson, J., Giorgi, S., Ringwood, J.V., 2015. Linear parametric hydrodynamic models for ocean wave energy converters identified from numerical wave tank experiments. Ocean Eng. 103, 31-39.

Eder, J., Bretl, J., Edwards, K., 2013. Empirical demonstration of acausal control strategies for wave energy converters. In: Proc. of 32nd International Conference on Ocean, Offshore and Arctic Engineering (OMAE). Nantes, France.

Evans, D., 1979. Some theoretical aspects of threedimensional wave-energy absorbers. In: First Symposium on Wave Energy Utilization, Gothenburg. Chalmers University of Technology.

Evans, D., 1981. Maximum wave-power absorption under motion constraints. Appl. Ocean Res. 3 (4), 200-203.
Falcão, A.F.d.O., 2007. Modelling and control of oscillatingbody wave energy converters with hydraulic power takeoff and gas accumulator. Ocean Eng. 34 (14-15), 2021-2032.

Falcão, A.F.O., Henriques, J.C.C., 2015. Effect of non-ideal power take-off efficiency on performance of single-and two-body reactively controlled wave energy converters. J. Ocean Eng. Mar. Energy 1 (3), 273-286.

Falnes, J., 1980. Radiation impedance matrix and optimum power absorption for interacting oscillators in surface waves. Appl. Ocean Res. 2 (2), 75-80.

Falnes, J., 1984. Wave-power absorption by an array of attenuators oscillating with unconstrained amplitudes. Appl. Ocean Res. 6 (1), 16-22.

Falnes, J., 2002. Ocean Waves and Oscillating Systems. Cambridge University Press, Cambridge, UK.

Falnes, J., Budal, K., 1982. Wave-power absorption by parallel rows of interacting oscillating bodies. Appl. Ocean Res. 4 (4), 194-207.

Fitzgerald, C., Thomas, G.P., 2007. A preliminary study of the optimal formation of an array of wave power devices. In: 7th European Wave and Tidal Energy Conference, EWTEC.

Folley, M., Whittaker, T., 2009. The control of wave energy converters using active bipolar damping. Proc. Inst. Mech. Eng. M: J. Eng. Marit. Environ. 223 (4), 479-487.

Fusco, F., Ringwood, J., 2010. Short-term wave forecasting for real-time control of wave energy converters. IEEE Trans. Sustainable Energy 1 (2), 99-106.

Fusco, F., Ringwood, J., 2012. A study of the prediction requirements in real-time control of wave energy converters. IEEE Trans. Sustainable Energy 3 (1), 176-184.

Fusco, F., Ringwood, J., 2013. A simple and effective realtime controller for wave energy converters. IEEE Trans. Sustainable Energy 4, 21-30.

Fusco, F., Ringwood, J., 2014. Hierarchical robust control of oscillating wave energy converters with uncertain dynamics. IEEE Trans. Sustainable Energy 5 (3), 958-966.

Garcia-Rosa, P., Ringwood, J., 2015. On the sensitivity of optimal wave energy device geometry to the energy maximising control system. IEEE Trans. Sustainable Energy 7 (1), 419-426.

Garcia-Rosa, P., Bacelli, G., Ringwood, J., 2015. Controlinformed optimal array layout for wave farms. IEEE Trans. Sustainable Energy 6 (2), 575-582.

Genest, R., Bonnefoy, F., Clément, A.H., Babarit, A., 2014. Effect of non-ideal power take-off on the energy absorption of a reactively controlled one degree of freedom wave energy converter. Appl. Ocean Res. $48,236-243$. 
Gilloteaux, J.C., Ringwood, J.V., 2010. Control-informed geometric optimisation of wave energy converters. In: Proc. of the 8th IFAC Conf. on Control Applications in Marine Systems (CAMS). pp. 399-404. Rostock, Germany.

Goldberg, D., 1989. Genetic Algorithms in Search, Optimization, and Machine Learning. Addison-Wesley, Reading, MA.

Hals, J., Falnes, J., Moan, T., 2011. A comparison of selected strategies for adaptive control of wave energy converters. J. Offshore Mech. Arct. Eng. 133 (3), 031101-031113.

Hals, J., Falnes, J., Moan, T., 2011. Constrained optimal control of a heaving buoy wave-energy converter. J. Offshore Mech. Arct. Eng. 133 (1), 011401.

Kracht, P., 2013. Wave prediction and its implementation on control systems of wave-energy converters. Frauenhofer IWES. EU MaRINet infrastructure access report.

Kurniawan, A., Moan, T., 2013. Optimal geometries for wave absorbers oscillating about a fixed axis. J. Oceanic Eng. 38 (1), 117-130.

Li, G., Belmont, M.R., 2014. Model predictive control of sea wave energy converters-Part I: A convex approach for the case of a single device. Renew. Energy 69, 453-463.

McCabe, A.P., 2013. Constrained optimization of the shape of a wave energy collector by genetic algorithm. Renew. Energy 51, 274-284.

McCabe, A.P., Aggidis, G.A., Widden, M.B., 2010. Optimizing the shape of a surge-and-pitch wave energy collector using a genetic algorithm. Renew. Energy 35 (12), 2767-2775.

Monk, K., Conley, D., Lopez, M., Zou, Q., 2013. Pneumatic power regulation by wave forecasting and real-time relief valve control for an OWC. In: 11th European Wave and Tidal Energy Conference (EWTEC).

Morari, M., Evanghelos, Z., 1989. Robust Process Control. Prentice-Hall, Inc., Upper Saddle River, NJ, USA

Nielsen, S.R., Zhou, Q., Kramer, M.M., Basu, B., Zhang, Z., 2013. Optimal control of nonlinear wave energy point converters. Ocean Eng. 72, 176-187.

Ochi, M.K., 1998. Ocean Waves: The Stochastic Approach. Cambridge University Press, Cambridge.

Ogilvie, T.F., 1964. Recent progress toward the understanding and prediction of ship motions. In: 5 th Symposium on Naval Hydrodynamics. pp. 2-5.

Paparella, F., Monk, K., Winands, V., Lopes, M., Conley, D., Ringwood, J.V., 2015. Up-wave and autoregressive methods for short-term wave forecasting for an oscillating water column. IEEE Trans. Sustainable Energy 6 (1), 171-178.
Price, A., Wallace, A., 2007. Non-linear methods for next wave estimation. In: 7th European Wave and Tidal Energy Conference, EWTEC.

Qiu, S.q., Ye, J.w., Wang, D.j., Liang, F.1., 2013. Experimental study on a pendulum wave energy converter. China Ocean Eng. 27 (3), 359-368.

Quine, B., Uhlmann, J., Durrant-Whyte, H., 1995. Implicit Jacobians for linearised state estimation in nonlinear systems. In: Proc. of the American Control Conf. pp. 1645-1646.

Retes, M.P., Merigaud, A., Gilloteaux, J.C., Ringwood, J.V., 2015. Nonlinear Froude-Krylov force modelling for two heaving wave energy point absorbers. In: 12th European Wave and Tidal Energy Conference (EWTEC).

Richter, M., Magana, M., Sawodny, O., Brekken, T., 2013. Nonlinear model predictive control of a point absorber wave energy converter. IEEE Trans. Sustainable Energy 4 (1), 118-126.

Ringwood, J., Bacelli, G., Fusco, F., 2014. Energy-maximizing control of wave-energy converters: the development of control system technology to optimize their operation. IEEE Control Syst. 34 (5), 30-55.

Scruggs, J., Lattanzio, S., Taflanidis, A., Cassidy, I., 2013. Optimal causal control of a wave energy converter in a random sea. Appl. Ocean Res. 42 (0), 1-15.

Shek, J., Macpherson, D., Mueller, M., 2008. Phase and amplitude control of a linear generator for wave energy conversion. In: 4th IET Conf. on Power Electronics, Machines and Drives (PEMD). pp. 66-70.

Taylor, C.J., Stables, M.A., Cross, P., Gunn, K., Aggidis, G.A., 2009. Linear and nonlinear modeling and control of a power take-off simulation for wave energy conversion. In: 8th European Wave and Tidal Energy Conference, EWTEC.

Teillant, B., Costello, R., Weber, J., Ringwood, J., 2012. Productivity and economic assessment of wave energy projects through operational simulations. Renew. Energy 48, 220-230.

Thomas, G., Evans, D., 1981. Arrays of three-dimensional wave-energy absorbers. J. Fluid Mech. 108, 67-88.

Zurkinden, A., Guerinel, M., Alves, M., Damkilde, L., 2013. Theoretical investigation of a wave energy system by applying reactive control using stochastic analysis of the wave state. In: Proc. 11th European Wave and Tidal Energy Conference. Aalborg, Denmark. 